\title{
Der Entwurf der deutschen Nachhaltigskeitsstrategie: Alter Wein oder großer Wurf?
}

Die Entwicklung einer deutschen Nachhaltigkeitsstrategie ist in die heiße Phase getreten. Seit Oktober führt der Staatssekretärsausschuss für nachhaltige Entwicklung (Green Cabinet) einen internetgestützten Dialogprozess (www.dialog-nachhaltigkeit.de). Der Rat für Nachhaltige Entwicklung veröffentlichte Ende November ein Dialogpapier. Schlieflich hat das Green Cabinet am 19. Dezember 2001 einen Strategieentwurf vorgelegt. Im März soll die Strategie vom Bundeskabinett beschlossen werden.

Mit den folgenden Expertisen soll ein Beitrag zum derzeit laufenden Diskussionsprozess geleistet werden. Nach einer kurzen Vorstellung von Eckpunkten des Entwurfs wird dieser von ExpertInnen aus Wissenschaft, Wirtschaft und Umweltverbänden kritisch diskutiert. Im Mittelpunkt stehen die drei Schwerpunkffelder Energie, Mobilität und Ernährung, Innovationspotenziale der Strategie sowie pointierte Auseinandersetzungen mit der StoBrichtung der Gesamtkonzeption.

\section{Eckpunkte des Entwurfs der Bundesregierung für eine nationale Nachhaltigkeitsstrategie}

Zusammengestellt von Jan Nill Als Ziel der Nachhaltigkeitsstrategie wird eine ausgewogene Balance zwischen den Bedürfnissen der heutigen Generation und den Lebensperspektiven künftiger Generationen angesehen (1). Es wird von einem breiten Nachhaltigkeitsverständnis ausgegangen: Nachhaltigkeit sei mehr als die Fortsetzung der Umweltpolitik mit anderen Mitteln, sie wird vielmehr als generelle Modernisierungsstrategie verstanden. Das entwickelte Leitbild geht von vier „Koordinaten“ aus:

- Generationengerechtigkeit,

- Lebensqualität,

Handlungsfeld Energie und Klimaschutz
- sozialer Zusammenhalt,

- internationale Verantwortung.

Auf dieser Basis wird ein Managementkonzept für eine nachhaltige Entwicklung entwickelt, das sich zusammensetzt aus

- 11 aus den Koordinaten abgeleiteten Managementregeln der Nachhaltigkeit,

- 21 Indikatoren und Zielen (siehe Kasten) sowie

- einem regelmäßigen Monitoring zur Erfolgskontrolle.

Die Indikatoren werden als Orientierungswerte für die staatlichen und gesellschaftlichen Akteure verstanden. Sie werden nur teilweise durch konkrete, quantifizierte Handlungsziele untersetzt. In der Regel wird dabei auf bereits bekannte Ziele zurückgegriffen, etwa hinsichtlich der Treibhausgasemissionen, der Verdopplung des Anteils der erneuerbaren Energien oder des angestrebten Anteils des Öko-Landbaus von 20 Prozent. Zumindest als offizielles Regierungsziel neu sind die Halbierung der Energie- und Robstoffintensität sowie die Verringerung der täglichen Zunahme der Siedlungs- und Verkehrsfläche von 130 auf 30 Hektar bis 2020. Die Höhe und der Zeithorizont der Ziele bleibt dabei teilweise deutlich

nicht übernommen. Die Chancen weiter Zeithorizonte, die kulturelle und materielle „Investitionszyklen“ übergreifen, bleiben insgesamt wenig genutzt.

Die Abkopplung von Zukunftsdiskursen von gegenwärtigen Interessenkonflikten kann in der politischen Praxis offensichtlich nur schwer geleistet werden. Genau diese Probleme und darauf ausgerichtete institutionelle Arrangements wären aber ein angemessener Gegenstand der Nachhaltigkeitsstrategie. Dem Drehbuch ein entsprechendes Inszenierungskonzept zu verpassen, das steht noch aus.

\section{Anmerkungen}

(1) Erster Bericht der Enquete-Kommission Nachhaltige Energieversorgung unter den Bedingungen der Globalisierung und der Liberalisierung. BT-Drucksache 14/7509, Berlin 2001, S. 21-72.

(2) Prognos/EWI: Die längerfristige Entwicklung der Energiemärkte im Zeichen von Wettbewerb und Umwelt. Untersuchung im Auftrag des BMWi. Basel 1999, S. 434.

\section{Der Autor}

Jan-Peter Voß ist wissenschaftlicher Mitarbeiter des Öko-Instituts.

Kontakt: Öko-Institut, Postfach 6226, 79038 Freiburg. Tel. 0761/ 45295-27, Fax 0761/475437, E-Mail: voss@oeko.de 
(c) 20I0 Authors; licensee IÖW and oekom verlag. This is an article distributed under the terms of the Creative Commons Attribution Non-Commercial No Derivates License (http://creativecommons.org/licenses/by-nc-nd/3.o/), which permits unrestricted use, distribution, and reproduction in any medium, provided the original work is properly cited. 